\title{
A comparison of the manufacture and mechanical performance of porous aluminium and aluminium syntactic foams made by vacuum-assisted casting
}

\author{
D Cheneler and A R Kennedy* \\ Materials Science Institute and Department of Engineering, Lancaster University , LA1 4YW, UK \\ *Corresponding author : a.kennedy3@lancaster.ac.uk
}

\begin{abstract}
This paper compares key aspects of the manufacture and mechanical performance of porous $\mathrm{Al}$, made by replication using salt beads, and structurally similar syntactic metal foams, made by replacing the salt with porous, expanded glass particles. Despite significant increases in stiffness, strength and energy absorbed, power law relationships between properties and relative density demonstrate that adding expanded glass particles is a less efficient route to increasing performance than increasing the metal fraction. That said, merit indices do indicate that $20 \%$ savings in mass are possible for stiff beams by substituting aluminium with syntactic metal foams. The manufacturing process for syntactic metal foams is shown to be simpler, driven by the lower thermal mass of the expanded glass particles and no requirement to dissolve a space holder from the structure. The balance of good performance and good manufacturability demonstrated herein, coupled with predicted low costs for raw material and manufacturing, highlights the scope for syntactic metal foams containing weak expanded glass particles to be researched and developed more widely.
\end{abstract}

Keywords: porous materials; casting; stress / strain measurements; finite element analysis 


\section{Introduction}

Porous aluminium and aluminium foams have the capability to deliver lightweight structures. With the additional benefits, beyond high specific stiffness and excellent energy absorption, of high temperature capability, sound and vibrational damping, their potential for considerable innovation in product design and performance, in particular for light weighting and improved user safety and comfort, is well documented [1, 2]. For example; Al foam components are being used as crash elements for a guardrail on the Massan-Chanwon Bridge in Korea, in the Combino tram in Budapest and the Sprinter Light Train (SLT) in Holland. There are also numerous ongoing trials of prototypes in the defence, rail, aerospace, leisure and shipbuilding sectors [3].

Despite these compelling cases, applications have barely expanded beyond those for high value products. The reason for this inertia is not performance but, despite lifecycle analysis showing overall cost savings, the price of the product at the point of sale is too high $[2,3]$. To understand this requires an appraisal of their methods of manufacture $[1,4]$. Closed pores are introduced into Al, either by bubbling gas through highly viscous Al melts, or by dispersing a gas-forming agent into molten $\mathrm{Al}$ or an $\mathrm{Al}$ powder compact, which is then melted. Large foam blocks can be cast to produce a semi-finished product. Creating shaped or sandwich-type products requires additional specialist equipment and processing steps, and careful process control is vital to achieve accurate products with acceptable reproducibility. Porous aluminium structures, with interconnected porosity, are commonly made by pressurised casting methods that either replicate the cavity left behind after removal of a reticulated polymer foam template from a plaster mould, or fill the gaps within a packed bed of sacrificial salt particles $[1,4-8]$. Specialist casting apparatus and protracted additional processing steps, to remove the mould or the salt particles, are required. These factors, and the cost of some raw materials, contribute to their high cost and are the main barrier to porous metals and metal foams becoming more mainstream.

Commercially viable foam products require the creation of uniform and reproducible porosity, and therefore properties, using affordable and scalable manufacturing processes that, where possible, combine integration of material production with component manufacture. Previous work [9] has shown the potential to adopt an alternative, novel approach to creating syntactic metal foams (SMFs) as alternatives to conventional metal foams and porous metals, by incorporating weak, highly porous, large, expanded glass particles (EGP). The manufacturing technology used to make these novel materials is essentially the same as that to produce 
microcellular aluminium [5-9], using a pressure differential to infiltrate a packed bed of particles with molten metal. The large particle size enables vacuum casting methods with simple, low cost apparatus, to be utilised.

This approach [9] is distinct from established research, as it did not use high strength cenosphere, or metal hollow sphere [10-14] additions. The rationale for this approach was given in [9], highlighting two key factors. Firstly, "traditional" Al syntactic foams containing hollow cenospheres (comprehensively reviewed in [15]), have higher densities $(1.5-1.9 \mathrm{~g} / \mathrm{cc})$ and lower porosities than metal foams and porous metals $(0.6-1.0 \mathrm{~g} / \mathrm{cc})$. This means that although the crush strength for these materials may be 10 times that for an equivalent metal foam or porous metal, the onset of densification occurs at significantly lower strains. Coupled with large load drops post yielding, caused by the fracture of the relatively strong cenospheres [9, 16-19], syntactic foams of this type are non-ideal energy absorbers. Secondly, Al syntactic foams are commonly made by isothermal molten metal infiltration of a bed of micron-sized hollow spheres. This process necessitates high infiltration pressures and large and costly processing apparatus.

This paper measures and compares key aspects of the mechanical performance of porous $\mathrm{Al}$, made by replication using salt beads, and syntactic metal foams (SMFs), made by replacing the salt used in the manufacturing process with EGP. Enhancements and detriments to performance are appraised in relation to the EGP addition and are contextualised against the benefits arising from the simpler manufacturing route.

\section{Methodology}

Expanded soda-lime glass particles (EGP) with a closed surface were used (supplied by OMYA). The approximate chemical composition (in wt.\%) of these particles is $69-73 \% \mathrm{SiO}_{2}, 11.5-13.5 \% \mathrm{Na}_{2} \mathrm{O}, 7.8-9.25 \% \mathrm{CaO}, 1-3 \% \mathrm{Al}_{2} \mathrm{O}_{3}$, $1.99-2.5 \% \mathrm{MgO}, 0.99-1.2 \% \mathrm{~K}_{2} \mathrm{O}$ and $0.49-0.59 \% \mathrm{Fe}_{2} \mathrm{O}_{3}$. The ellipsiodal beads were sieved between 2 and $3 \mathrm{~mm}$ in diameter, have an average density of $0.35 \mathrm{~g} / \mathrm{cc}$, with an estimated porosity of $80-85 \%$ [20]. Their morphology is shown in Figure 1a. Approximately 15g of beads were poured into a stainless steel metal mould, coated with graphite paint and perforated at the base, containing a cavity approximately $34 \mathrm{~mm}$ in diameter and $120 \mathrm{~mm}$ long. The packing density was altered up to a maximum of approximately $69 \%$, by applying varying degrees of tapping after filling. Moulds were placed into a preheated muffle furnace, at $300^{\circ} \mathrm{C}$, and then infiltrated under vacuum using a Per-Cast Vacuum Casting machine (HS Walsh, UK [21]). Using this method, a reduced pressure is applied to the base of the perforated mould, whilst the upper surface is exposed to the atmosphere. The infiltrant was an LM6 grade aluminium alloy, sourced from Norton Aluminium, heated to $700^{\circ} \mathrm{C}$. The specification for the chemical 
composition for this alloy is $10-13 \mathrm{wt} . \% \mathrm{Si}$, with all other elements present below $0.6 \mathrm{wt} . \%$ (for fuller detail of the composition specification see [22]). The vacuum level was varied between 0.25 and 0.9 bar in order to alter the degree of infiltration and the density of the final component. Castings were solidified in the apparatus, without additional measures to control the direction or speed of solidification. Cast syntactic foam samples were machined to $32 \mathrm{~mm}$ in diameter and $40 \mathrm{~mm}$ in length. Porous aluminium alloy samples were made by replacing the EPG particles with $92 \mathrm{~g}$ of near-spherical salt beads (Hydrosoft granular, Peacock Salt, UK) and preheating the mould and beads to $600^{\circ} \mathrm{C}$. The morphology of these salt $(\mathrm{NaCl})$ beads, which were sieved to the same size range as for the EGP, is shown in Figure 1b. After infiltration, the salt was removed by dissolution in water, held between 30 $35^{\circ} \mathrm{C}$, for up to 24 hours, to create the porosity. Samples were characterised using optical and scanning electron microscopy (SEM) along with X-ray micro-computed tomography (CT).

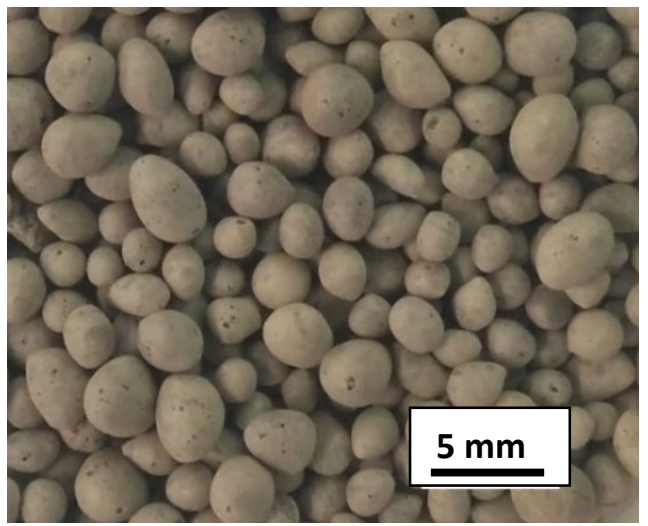

a)

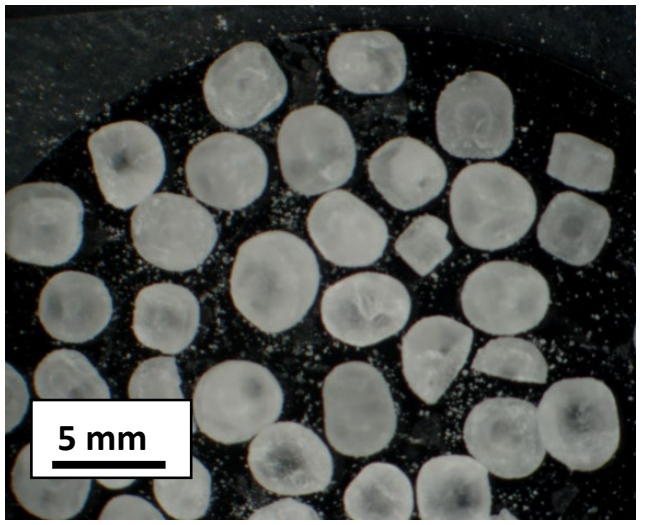

b)

Figure 1 Morphology of a) the expanded glass particles (EGP) and b) the salt beads

Quasi-static compression tests were performed at a constant displacement rate of $0.4 \mathrm{~mm} / \mathrm{s}$. A pre-calibrated linear-variable differential transformer (LVDT), with an effective resolution, based on the signal conditioning hardware, of less than $0.1 \mu \mathrm{m}$, was used to accurately measure the displacement of the sample. Stiffness was measured by averaging over 4 loading and unloading cycles between 25 and $75 \%$ of the yield stress. The crush strength was determined from the stress at a strain of 0.25 , to help simplify the interpretation of the complex crushing behaviour close to the yield point, and both the energy absorbed and the energy absorption efficiency were measured for strains of 0.25 and 0.5 . 


\section{Results and discussion}

\section{Processing and structure}

Syntactic foams were successfully produced using mould preheating temperatures that are typical of those used in metal die casting; the low thermal mass of the EGP facilitates this. This is in stark contrast to the salt beads, where their high thermal mass (6 times higher in $\mathrm{J} / \mathrm{K}-\mathrm{NaCl}$ and glass have similar specific heat capacities) necessitates impractically high mould preheating (to $600^{\circ} \mathrm{C}$ ) to avoid premature freezing of the metal before infiltration is complete. For the EGP beds, preheated to $300^{\circ} \mathrm{C}$, infiltration took less than $5 \mathrm{~s}$, whilst the casting took more than $40 \mathrm{~s}$ to freeze. The additional surface area created by the beads, and the reduced metal content, causes the casting to solidify faster; it takes approximately $70 \mathrm{~s}$ for the melt to freeze in an "empty" mould. Infiltration is rapid, even for the very modest pressure differences applied, owing to the high permeability of packed beds containing 2-3 mm diameter spheres [23]. Similar performance, in terms of infiltration rate, with slower melt freezing (approximately $130 \mathrm{~s}$ ), was achieved for salt beads preheated to $600^{\circ} \mathrm{C}$, but for preheating temperatures below the melting point of the metal, and for small pressure differences, infiltration was not complete before freezing occurred.

By considering a maximum (measured) EGP packing fraction of 0.69 , the density of the resulting syntactic metal foam (SMF) would be $1.08 \mathrm{~g} / \mathrm{cc}$, given complete infiltration of the packed bed. For loose packing, with a (measured) packing fraction of 0.64 , the maximum density achievable would be $1.20 \mathrm{~g} / \mathrm{cc}$. By varying the packing conditions and the pressure difference, from 0.25 to 0.9 bar, syntactic foam samples with a range of different densities, from 1.00 to $1.19 \mathrm{~g} / \mathrm{cc}$, were produced. For a given set of packing conditions and infiltration pressures, samples with densities varying by no more than $\pm 0.01 \mathrm{~g} / \mathrm{cc}$ were produced, demonstrating good reproducibility. The porosity was varied in a similar manner for the porous (salt route) samples, where in this case the packing fraction for the salt particles, with slightly different shape and size distribution to the EGP, varied between 0.64 and 0.73 , leading to samples that varied in density between 0.64 and $0.96 \mathrm{~g} / \mathrm{cc}$. Figure 2 compares the macrostructures for porous metal and syntactic foam castings that have been machined, ready for compression testing. A similar packing behaviour is apparent for the two "space filler" materials. By leaving the EGP in the cast structure, the mass (and therefore density) of these "equivalent" structures, with similar metal fractions will always be higher. 


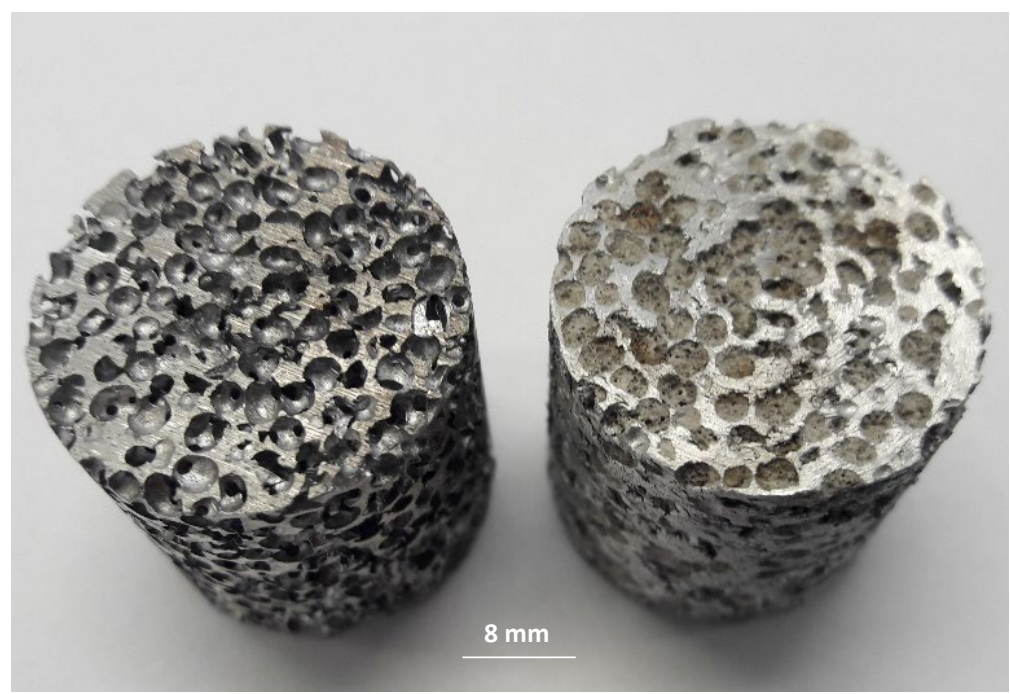

Figure 2 Images showing (left) porous metal and (right) syntactic metal foam samples in the machined condition, prior to compression testing.

Figure 3 shows CT images of the cross section of a syntactic foam casting. It can be seen that the EGP are well packed and distributed throughout, confirming that the process is capable of producing an even distribution of porosity. Of course, similar good distributions of porosity are observed for porous structures made from salt beads $[5,8,9,24-26]$, as the packed structures and sizes for the "space filler" materials are similar. It has been remarked [27], that the structure of porous metals made by replication, for example features such as the strut cross section and the relative distribution of metal in the struts and at the nodes, varies with metal fraction. It is reasonable to assume that the two materials compared in this study have, for the same metal fraction, similar structures, allowing an accurate appraisal of the influence of the addition of EGP. Figure 3 also shows that the EGP contain pores with an average diameter between 100-200 $\mu \mathrm{m}$, separated by thin walls a few $\mu \mathrm{m}$ thick. This higher magnification image shows that for the case presented, using the minimum pressure differential of 0.25 bar, only partial infiltration of the contact regions between the EGP occurs, creating additional void space (labelled in this figure) containing air at low pressure.

The relationship between the capillary pressure and curvature for a pendular ring of fluid bridging contacting spheres, is given by the Young-Laplace equation [28]. For the case presented here, a toroidal approximation is adequate [28], and $r_{1}$ is well approximated by the neck radius and $r_{2}$ by the perpendicular profile radius for the liquid-vapour interface. $\gamma_{\mathrm{LV}}$ is the liquid-vapour interfacial tension. 


$$
\Delta P=\gamma_{L V}\left(\frac{1}{r_{1}}-\frac{1}{r_{2}}\right)
$$

Figure 4a marks the radii for the two curvatures referred to in Equation 1, superimposed over a CT image of an EGP preform infiltrated with a 0.25 bar pressure differential. The circles are of the same radius, $100 \mu \mathrm{m}$, and indicate that the curvature of the liquid front is broadly similar at the different contact points. Despite measurements for $r_{1}$ and $r_{2}$ being inaccurate, owing to sectioning effects, and the toroidal approximation of Equation 1 being limited, given that particles are non-spherical and they range in size and shape, Figure 4 clearly shows that an increase in pressure differential decreases the curvatures, which will lead to a small reduction in porosity. Figures 3 and 4 also reinforce the simple model developed in [8], which describes the geometry of "windows" formed between contacting pores in porous metals made by salt bead replication. The void region defines the diameter of the window that spans neighbouring pores, with lower pressure differences clearly resulting in larger windows.

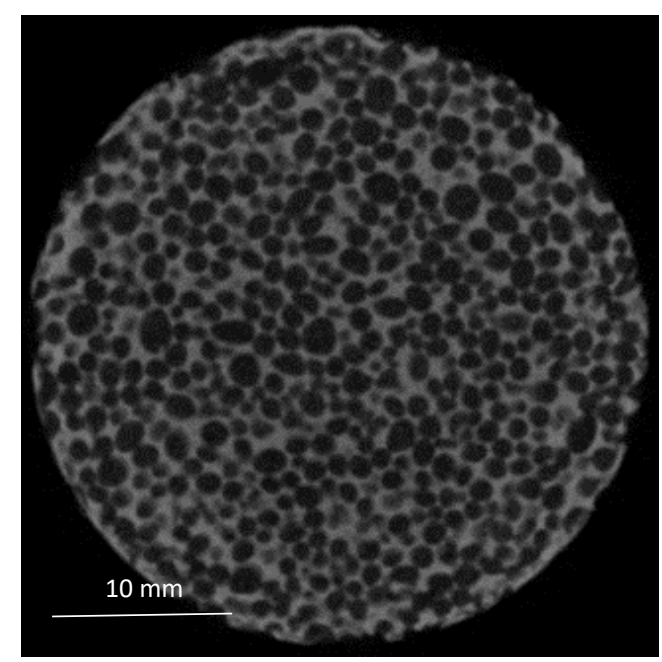

a)

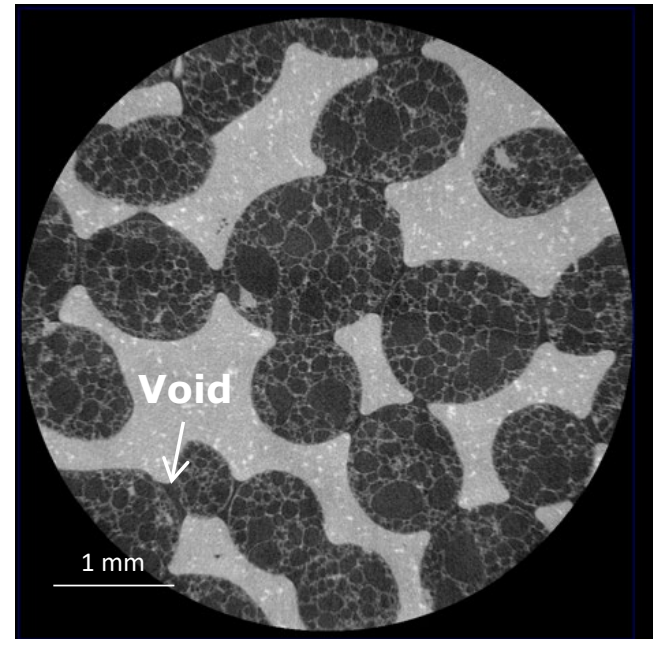

b)

Figure 3. CT images of the structure of Al-Si - EGP syntactic foams at a) low and b) high magnification. 


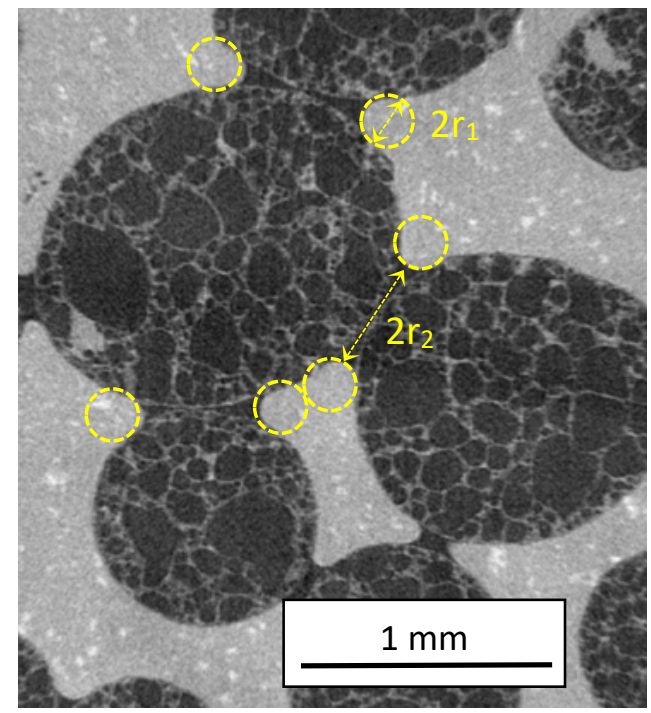

a)

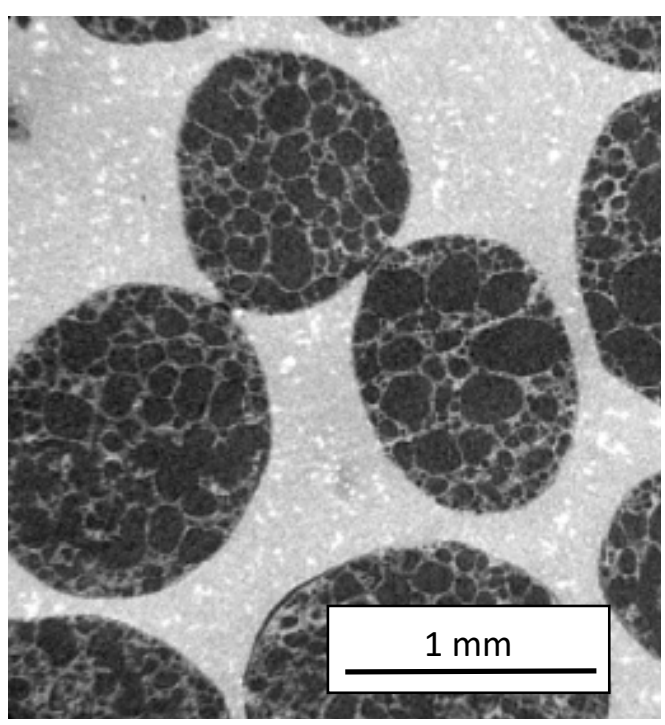

b)

Figure 4. CT images illustrating the interface curvatures, comparing EGP samples infiltrated at a) the minimum and b) the maximum pressure differentials.

As reported in a previous study [9] and again confirmed by optical and scanning electron microscopy in this work, the matrix microstructure for infiltrated samples containing EGP remained characteristic of a near-eutectic Al-Si alloy and energy dispersive X-ray analysis of the matrix did not reveal the presence of $\mathrm{Na}, \mathrm{Ca}$ or $\mathrm{Mg}$ that might have leached from the EGP. Un-reacted interfaces are to be expected given the less than 1 min exposure times in the molten state. The EGP remain intact during the infiltration process and no penetration by molten Al-Si was observed, as evidenced by images in Figures 3 and 4.

\section{Mechanical performance}

Figure 5 shows typical stress-strain curves for syntactic metal foam and porous metal samples. The inset image is a typical stress - strain curve for low strains, showing the linear and overlapping loading / unloading cycles used to determine the modulus. The data chosen for this comparison allow consideration of roughly equivalent metal fractions, and hence, as discussed earlier, similar structures for the metal phase. For example, for a foam density of $0.83 \mathrm{~g} / \mathrm{cc}$, the relative density is approximately 0.31 . If this metal fraction remains constant and the porosity is filled with EGP with a density of $0.35 \mathrm{~g} / \mathrm{cc}$, the corresponding syntactic foam density will be approximately 1.07 $\mathrm{g} / \mathrm{cc}$. 
Stress-strain curves for the porous metal samples are typical of those in the literature (reviewed in [1]). After yielding, the stress is nearly constant, rising gradually with strain. For traditional porous materials with high porosity and thin cell walls, typically with relative densities $<0.2$ (for Al, corresponding to a density $<0.54 \mathrm{~g} / \mathrm{cc}$ ) [29], cell wall buckling is the main deformation mechanism that facilitates compression under constant stress. For lower porosities, as the cell walls thicken, the formation of plastic hinges is less likely. In such specimens an increased level of work hardening is observed and the dominant failure mode tends to be cell wall shearing [29]. Using a simple expression in [1], the strain for the onset of densification, where stress starts to increase markedly with further straining, for a porous metal with a density of $0.83 \mathrm{~g} / \mathrm{cc}$, is estimated to be in the range of $0.5-0.56$. This prediction is broadly in keeping with the behaviour observed in Figure 5 and that reported in [29].

The stress-strain curves for the syntactic metal foam were found to take two distinct forms (labelled as Type I and II). Regardless of type, the stress-strain curves for the SMFs undulate to a greater degree than those for porous metals. For Type I, the stress increases considerably with strain, at strains exceeding 0.3 . Although not entirely conclusive, this behaviour was more commonly observed in syntactic foams with higher porosity, correlating with densities < approximately $1.1 \mathrm{~g} / \mathrm{cc}$. The predicted strain for the onset of densification for a syntactic metal foam with a density of $1.07 \mathrm{~g} / \mathrm{cc}$ is in the range of $0.38-0.42$, similar to that observed in Figure 5 for Type I SMFs. Type II behaviour is observed in SMFs with lower porosity and is characterised by a nearly flat stress - strain curve over the strain interval examined. This behaviour resembles that observed in $[30,31]$ for similar syntactic metal foams. In these studies, rapid increases in stress with strain were only observed at very high strains $(>0.7)$, strains that are in excess of the maximum theoretical densification strain, that which is calculated based on the elimination of all the available void space (circa. 0.6). 


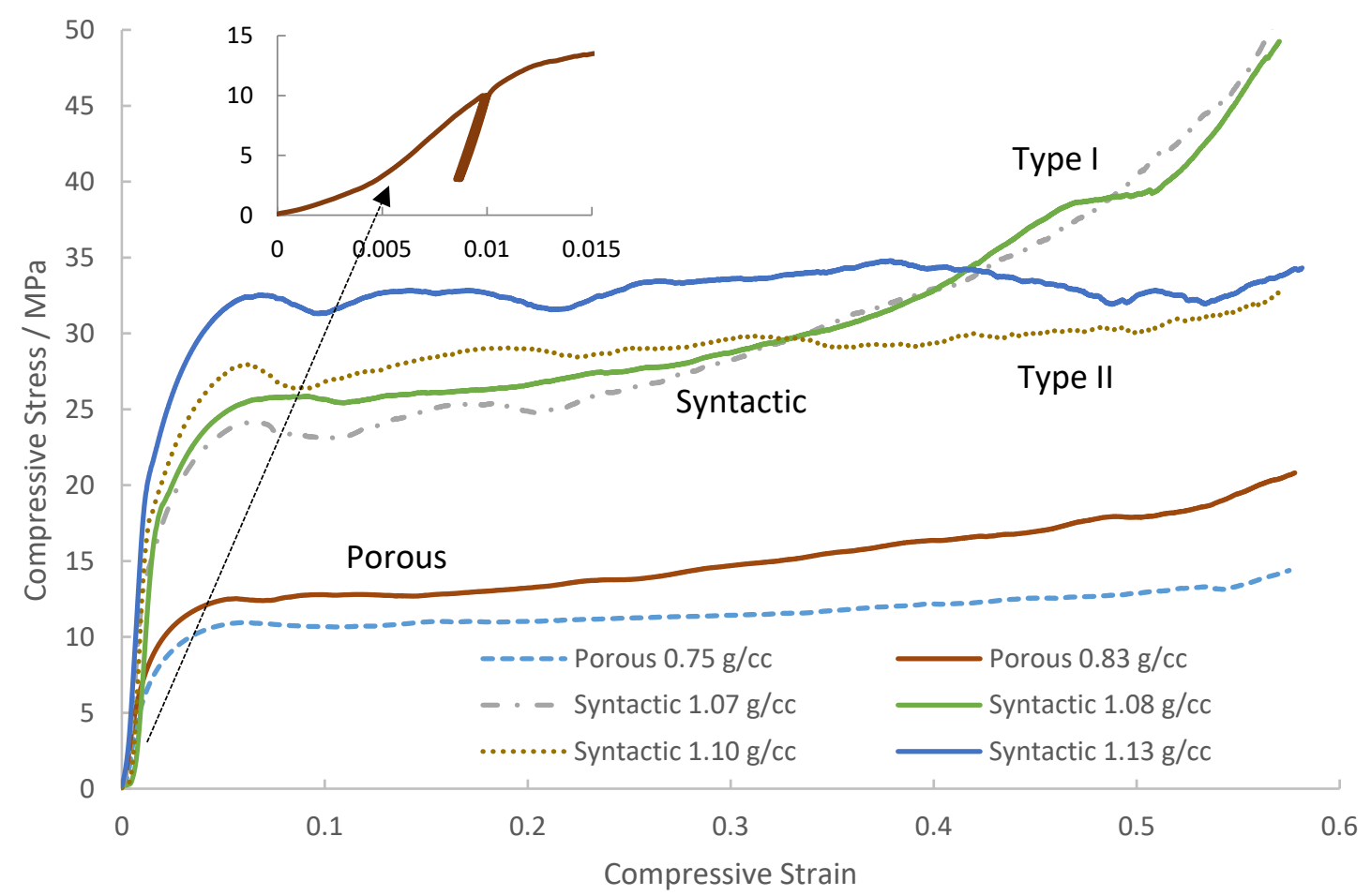

Figure 5 Typical stress - strain behaviour for porous metals and syntactic foams with roughly equivalent metal fractions (inset: stress-strain curve at low strains, showing the loading/unloading cycles).

Figure 6 shows stiffness data for the $40+$ samples tested. The values reported are an average of the 4 loading / unloading cycles, which are typically within $2 \%$ of the mean. Measurement errors for stress and strain are typically less than $0.5 \%$. Total errors are smaller than the symbols used to represent them and have been omitted for clarity throughout. As expected, the trend for an increase in stiffness with increasing density is observed in both materials. Both materials follow a power law dependence, with exponent approaching 3 , with a slightly higher value for the syntactic metal foam. It should be noted that stiffness data for the porous material agree well with those reported for microcellular Al made by salt bead replication, 1-4 GPa, for samples with densities in the range of $0.54-0.81 \mathrm{~g} / \mathrm{cc}$, as reported in $[6,32,33]$. The stiffness values for the syntactic foams are roughly double those reported for similar materials in [30,31], where measurements were made without either the use of direct strain measurement, for example with an LVDT, or compensation for the compliance of the testing machine. Data from [24] show that strain calculation from the crosshead displacement, without compensation for the machine compliance, can reduce the measured stiffness by as much as $40 \%$, and this could account for the differences observed. Figure 6 also contains what has been termed "modified" data for the porous metal, translating the 
density, as outlined in the earlier example, to that which would include filling the available porosity with EGP. This analysis allows a clearer estimate of the contribution of the EGP to the stiffness of similar metal structures across the full data set. The presence of the EGP increases the stiffness of the porous metal structure by between 2 and $3 \mathrm{GPa}$.

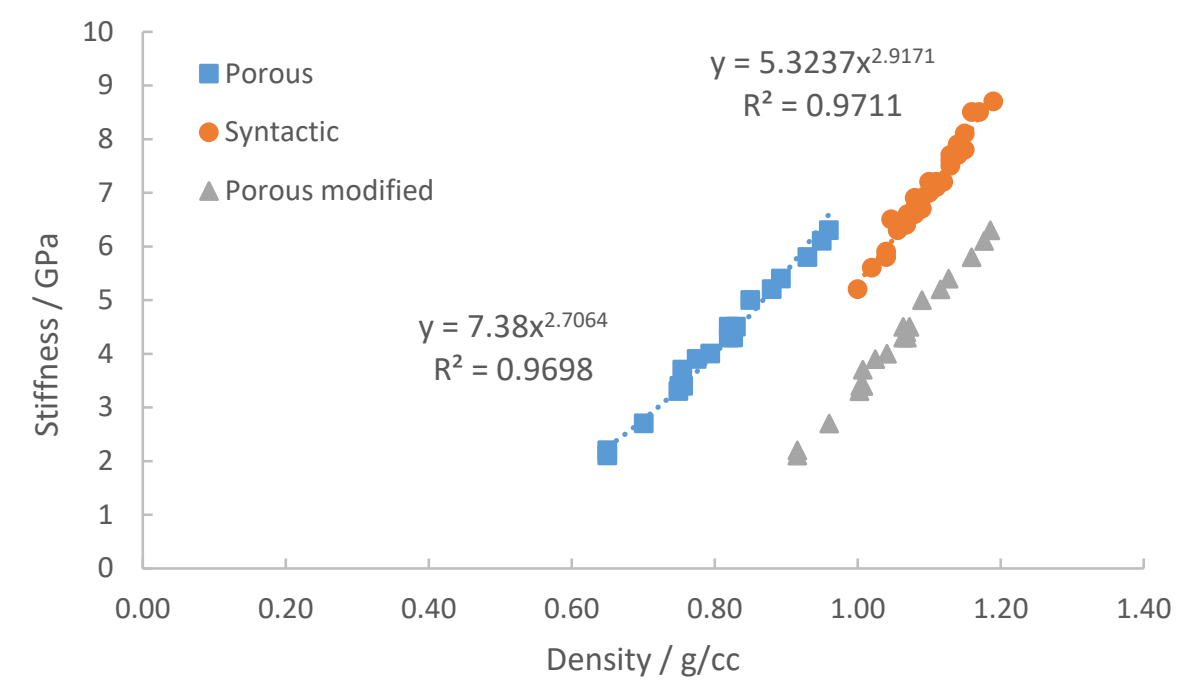

Figure 6 Stiffness as a function of density for syntactic metal foam and porous metal samples.

Figure 7 presents crush strength data. As expected, the trend for an increase in stress at 0.25 strain with increasing density is observed for both materials. As the fits to the curves in Figure 7 demonstrate, both materials follow a power law dependence with exponent close to 3 , once more with a higher value for the syntactic metal foam. The "modified" data show that, on average, EGP addition increases the crush strength across the whole data set by approximately $13 \mathrm{MPa}$.

The power law exponents describing both the influence of density on stiffness and strength are higher than those reported for "perfect" structures (2 and 1.5 respectively [1]). Modelling and experimentation [27, 34] have shown that exponents close to 3 might be expected, for both the stiffness and strength expressions, for "imperfect" structures made by salt bead replication. It is proposed that foam structures of the type reported herein are inefficient, with a large amount of material concentrated at the nodes, where the struts meet, which adds mass but contributes little to the strength [32]. The architecture of these structures also changes with metal fraction as both the number of struts and their cross section varies [33]. These factors lead to a greater than expected decrease in 
stiffness and strength as the relative density decreases and thus a higher value for their power law exponents. The addition of EGP further increase this inefficiency, a result of additional mass being distributed away from the cell struts, manifested by a higher value for the power law exponent.

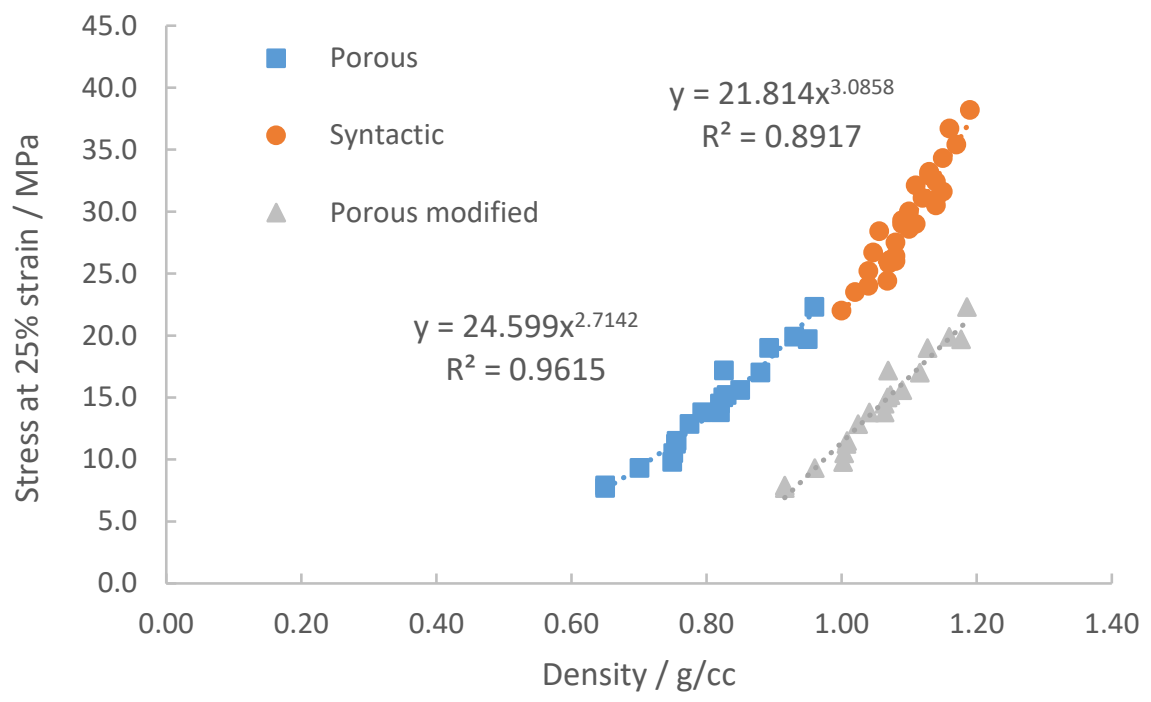

Figure 7 Crush strength (for 0.25 strain) as a function of density for syntactic metal foam and porous metal samples.

Porous metals are considered to have good potential for stiff and strong, lightweight panels. To quantify this, the merit indices for light, stiff and strong beams can be determined by calculation of $E^{1 / 3 /} / \rho$ and $\sigma^{1 / 2} / \rho$, and it is reasonable to assume that the values for the compressive stiffness and strength for these structures are no lower than those values in tension [1]. As the beam mass, for a given deflection, is inversely proportional to the merit index, maximising this, maximises the weight saving. Values for the porous Al structures in this study are, on average, 2.0 and 4.2 respectively, and for SMFs, 1.8 and 5.0 respectively. Merit indices for the equivalent Al alloy are 1.5 and 3.6, showing large mass savings for both porous materials. Power law exponents close to 3 mean that the stiffness merit indices are only very weakly dependent upon the density, but that for the strength, M decreases with decreasing density. When comparing structures with similar densities, the porous metal, with the more efficient structure, offers the greater potential for weight saving for both strong and stiff beams.

Further insight into the deformation of the metal structures can be gleaned through finite element (FE) modelling (see Supplementary Information for the modelling approach and raw data). In short, the model compares two 
identical cells, one with holes, the other containing a material (EGP) with an assumed stiffness of $1.5 \mathrm{GPa}$ (estimated based on a scaling law [1]) and a yield strength of $5 \mathrm{MPa}$ [30]. The matrix stiffness and strength were assumed to be $71 \mathrm{GPa}$ and $92 \mathrm{MPa}[35,36]$ respectively. Compression was applied in the vertical plane. Figures 8 and 9 show the von Mises stresses for strains of 0.0005 and 0.02 for the porous aluminium and SMF structures respectively (taken as points significantly below and above the point of yielding). The wireframe structure shows the original, undeformed structure, from which it is apparent that deformation is localised at the top of the cell, for both materials. Figure 8 indicates that deformation initiates at the narrowest point of the struts, where the equivalent or von Mises stress is beyond the yield strength of aluminium. Simulations show that the maximum shear stress also occurs at these same locations, lending support to the subsequent deformation and localised failure of these thick struts within the porous metal structure occurring by shearing rather than buckling [29]. Figure 9 shows that a very similar pattern of deformation and localised stress develops in the SMF structure containing EGP. The areas where yielding initiates, and where the maximum shear stress occurs, remain the same as for the porous structure. Yielding still originates in the matrix, despite the EGPs having a much lower yield strength. Figure 10b shows that despite the matrix being loaded beyond its yield point, the EGP are only just starting to be loaded beyond their yield stress. This prediction, of matrix yielding before yield, or more correctly fracture, of the EGP, is supported by experimental observations made using acoustic emission [9].

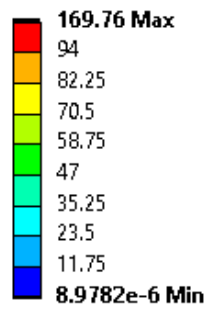

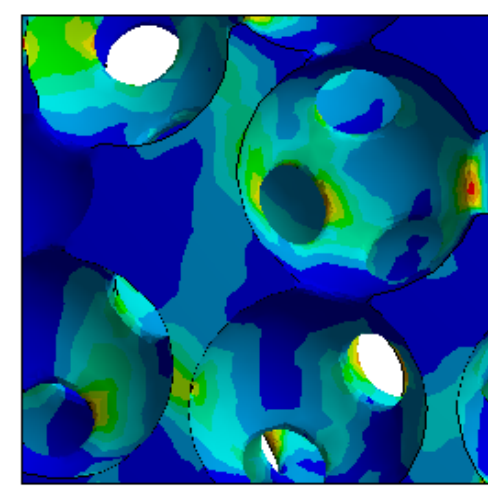

a)

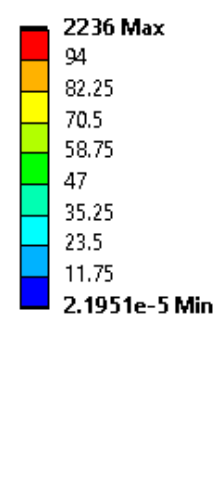

b)

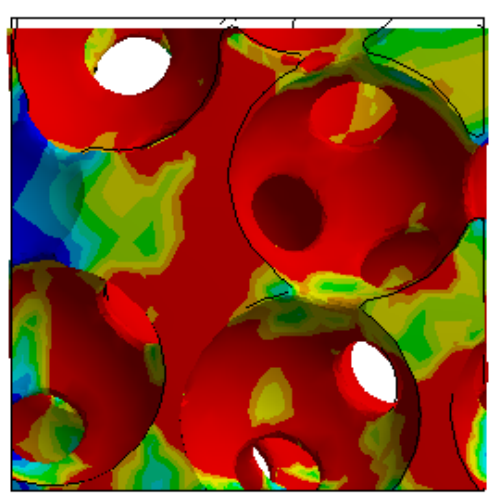

Figure 8 Equivalent, or von-Mises, stress within the aluminium matrix, a) at 0.0005 effective normal strain and b) at 0.02 effective normal strain. Units in MPa. Wireframe denotes undeformed geometry. Top contour denotes stress above yield strength of aluminium matrix. 


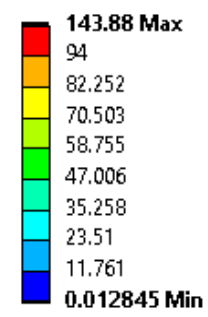

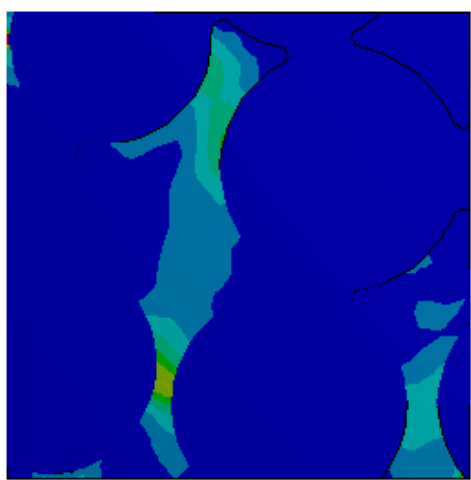

a)

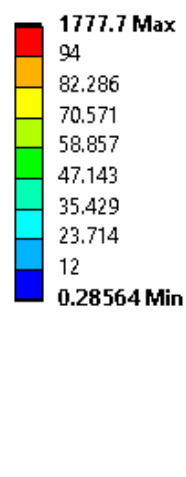

b)

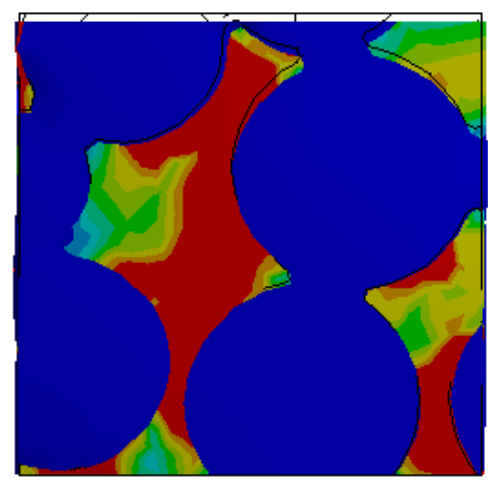

b)

Figure 9 Equivalent, or von-Mises, stress within the composite, a) at 0.0005 effective normal strain and b) at 0.02 effective normal strain. Units in MPa. Wireframe denotes undeformed geometry. Top contour denotes stress above yield strength of aluminium matrix.

Simulations estimate the stiffness of the porous aluminium and SMF to be 9.3 and 11.6 GPa respectively, higher than the 6.3 and 8.7 GPa measured for samples with near-equivalent densities. Whilst the model over-estimates the stiffness, most likely owing to the limited scale and defect-free nature of the idealised modelled structure and the contact conditions applied, the difference in stiffness between the SMF and porous structure is very similar to that for the experimental measurements, supporting the hypothesis that the EGP are providing some, albeit small, "reinforcement" to the matrix. Simulated flow stresses at 0.02 strain of 29.5 and $35.5 \mathrm{MPa}$, for the idealised porous and SMF structures respectively, are again higher than those measured (19 and $33 \mathrm{MPa})$, but the difference in values between structures is much smaller for the simulations. As the extent of EGP yielding is low, the simplified approach to modelling their post-yield deformation is less likely to contribute to the inaccuracy than estimations of the yield strength of the matrix and ignoring any change in this owing to the presence of the EGP.
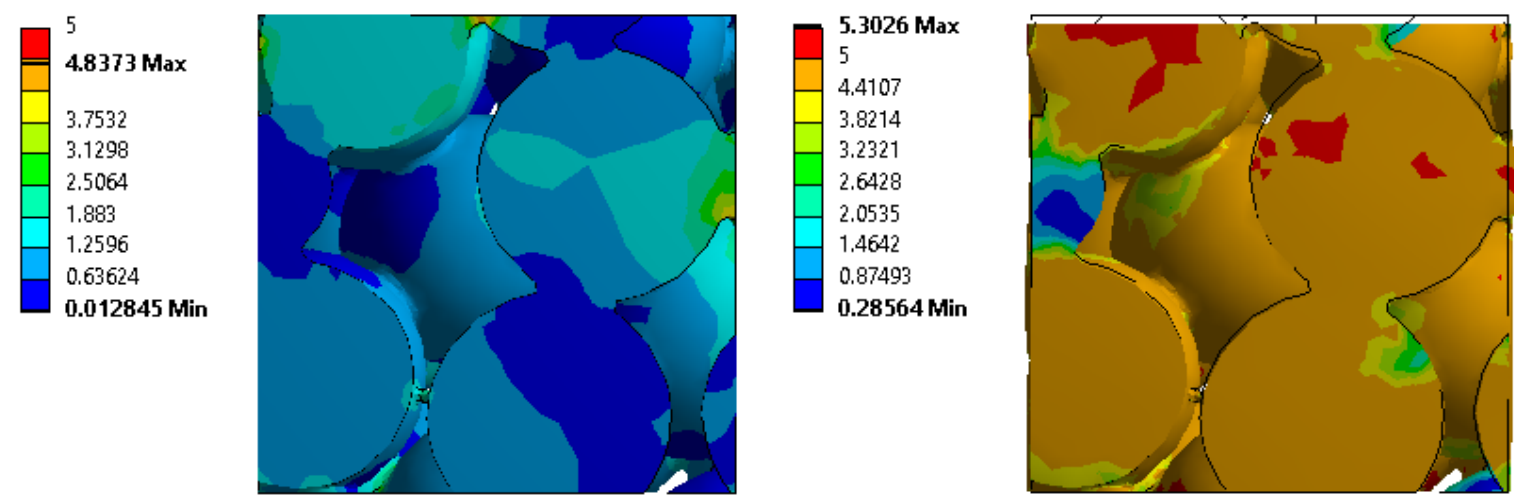
Figure 10 Equivalent, or von-Mises, stress within the composite with the aluminium matrix hidden showing just the stress in the EGPs, a) at 0.0005 effective normal strain and b) at 0.02 effective normal strain. Units in MPa.

Wireframe denotes undeformed geometry. Top contour denotes stress above yield strength of EGPs.

Figure 11 shows the energy absorbed per unit volume (the integral of stress with strain, from zero strain to a given upper limit), up to a strain of 0.25 , for both sets of materials. Comparison with the modified porous data indicates that the absorbed energy per unit volume increases, on average, by between $4-6 \mathrm{MJ} / \mathrm{m}^{3}$ with the addition of EGP. Values are in close agreement with the literature for both porous structures $\left(2-4 \mathrm{MJ} / \mathrm{m}^{3}[29]\right)$ and syntactic metal foams (5-6 MJ/m³ [30], estimated from data up to 0.5 strain), for similar alloys with similar densities. Dividing by the density gives the energy absorbed per unit mass, yielding values between approximately $3-5 \mathrm{~kJ} / \mathrm{kg}$ for the porous metal and $5-7 \mathrm{~kJ} / \mathrm{kg}$ for the syntactic foam. The significant increase in both metrics for the syntactic foam are a result of the large increase in the crush strength for this material (see Figure 7). Figure 11 shows that energy absorption follows a power law dependence upon the density since, as was commented previously, the porous structures are density dependent. The power law exponent is again higher for the syntactic foam, indicating that adding EGP is a less efficient route to increasing energy absorption, both per unit volume and mass, than increasing the metal fraction.

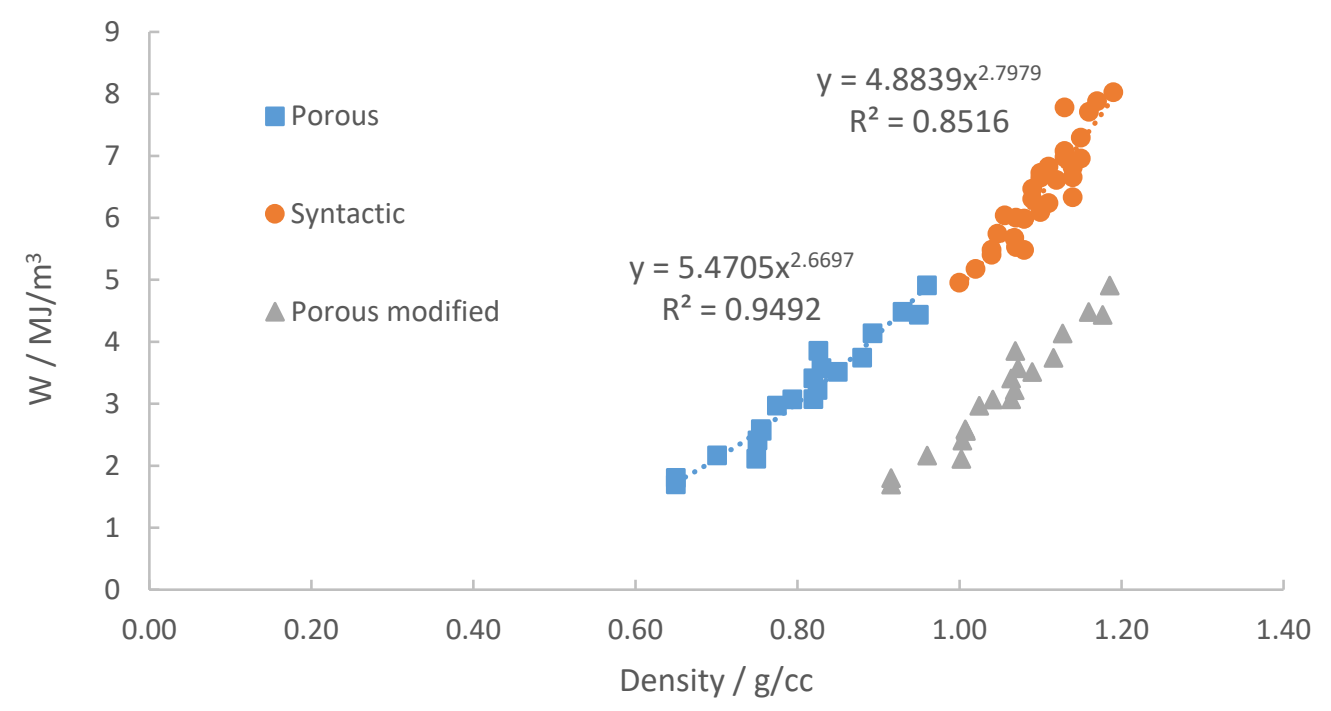


Figure 11 Energy absorbed per unit volume, up to a strain of 0.25 , for porous $\mathrm{Al}$ and syntactic foams, plotted as a function of density.

Figure 12 shows the energy absorption efficiency for the full data set, plotted for strains of 0.25 and 0.5 , calculated from the area under the stress strain curve, divided by the product of the stress and strain at the target strain (the perfect flat stress - strain behaviour). For 0.25 strain, all values are high, close to $90 \%$, highlighting the excellent efficiency for both these materials, a result of the flat stress-strain response at lower strains, as was evident in Figure 5. For the porous metals, at 0.5 strain, the efficiency drops slightly, a result of the slight increase in stress with strain, typified in Figure 5. No strong influence of porosity was observed, over the range considered, but it might be expected that the efficiency would decrease for lower porosities, as the degree of work hardening increases [29] and the strain for the onset of densification decreases. The syntactic metal foams display a bifurcation in behaviour, which relates to the two different types of mechanical response identified in Figure 5. In those samples with a flat stress - strain curve, Type II, the absorption efficiency remains high at higher strains. For Type I SMFs, those with lower density, or higher porosity, the absorption efficiency decreases significantly when considered over a 0.5 strain interval.

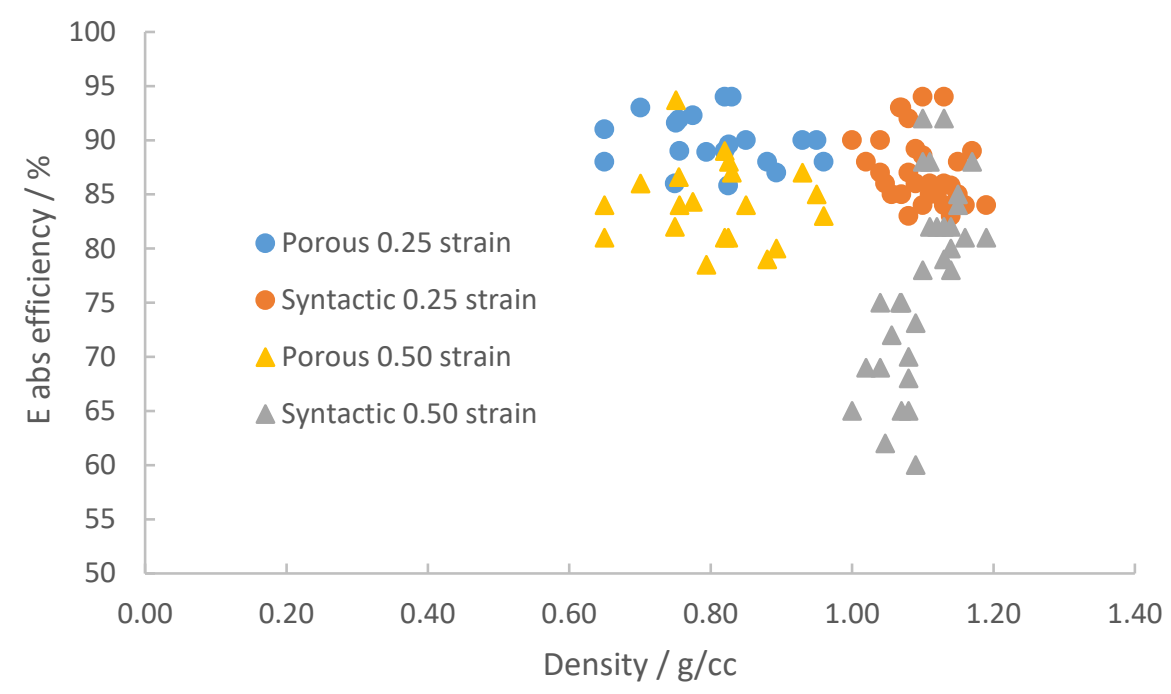

Figure 12 Energy absorption efficiency for syntactic metal foams and porous metals.

Figure 13 shows this more clearly, displaying the energy absorption efficiency, for selected samples, as a function of strain, over the whole interval measured. For all these samples, the efficiency is a maximum for strains between 
0.2 and 0.3 , with values between 85 and 95\%. For materials with a largely flat stress-strain response (porous and syntactic Type II), this efficiency remains high, until testing was stopped at just below 0.6 strain. For syntactic Type I materials, which show stress increasing at higher strains, the overall efficiency can drop dramatically, to as low as $60 \%$ at 0.5 strain.

The difference in performance is related to the fracture behaviour during compression, shown through example images superimposed on Figure 13. Syntactic foams with higher porosity or lower density (Type I), in general, compress in a fashion similar to porous metals [29], but densify at lower strains. Beyond the onset of densification, the absorption efficiency decreases progressively with increasing strain. Figure 13 shows that these samples remain largely intact after compression; the sample presented had an energy absorption efficiency of $63 \%$ at 0.5 strain. Syntactic metal foam samples with higher densities or lower porosities, crush at strains above approximately 0.3 with continual fragmentation. Barrelling, observed for Type I SMFs, is avoided. The extent of disintegration is clear in Figure 13, where fragments of metal and EGP debris are apparent for a syntactic metal foam sample with an energy absorption efficiency of $90 \%$ at 0.5 strain. This behaviour is similar to that for similar materials in $[30,31]$ where extensive fragmentation was observed at strains above 0.3 .

The exact transition point between Type I and II behaviour in this study is unclear, especially when considered in terms of the sample density; the same density can be achieved for samples with different combinations of EGP packing density and infiltration conditions. It is proposed that Type I behaviour occurs in samples with higher porosity, where the EGP packing factor is high and low infiltration pressure differences create significant interparticle voids (as was observed in Figure 3). The introduction of this higher level of dispersed inter-particle porosity seems to promote a layer-by-layer collapse process, rather than the formation of catastrophic shear bands. The use of vacuum, rather than positive pressure $[30,31]$, to drive the infiltration process, appears to favour Type I behaviour. 


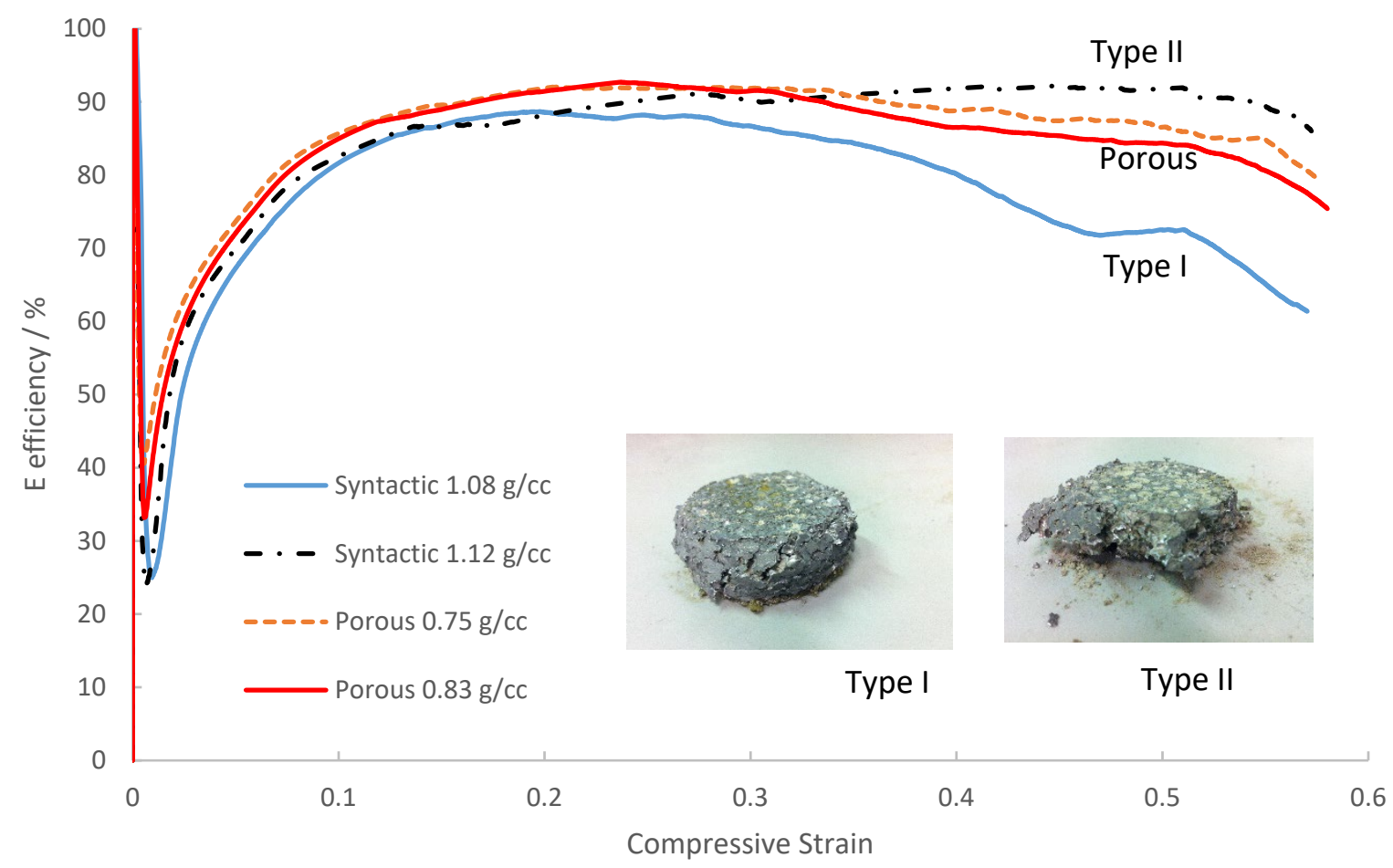

Figure 13 Energy efficiency as a function of strain for syntactic metal foams and porous metals with equivalent metal fractions. Images for samples after compression are also presented.

\section{Summary and conclusions}

The addition of weak expanded glass particles (EGP) increases the stiffness of porous metal structures by approximately $50 \%$, the strength by $100 \%$, the energy absorbed per unit volume by $100 \%$ and by mass, by $50 \%$, albeit with an increase in density. Each of these aforementioned properties can be related to the relative density of the resulting syntactic metal foam (SMF) or the porous metal structure by a power law. The power law exponent is, however, higher for the syntactic foam, indicating that adding EGP is a less efficient route to increasing performance than increasing the metal fraction. Despite poorer structural efficiency compared to porous metal structures, merit indices indicate that $20 \%$ savings in mass are possible for stiff beams by substituting aluminium with syntactic metal foams.

There are clear advantages to the manufacturing process for syntactic metal foams over those for porous metals. The low thermal mass of the EGP enables the manufacture of SMF parts using conventional vacuum or low pressure die casting methods, without the need for excessive pre-heating of inserts or tooling. This, combined 
with the low cost of the EGP (typically $1 / 20^{\text {th }}$ that for $\mathrm{Al}$ and $1 / 6^{\text {th }}$ that for salt, for the same volume) and no requirement to dissolve a space holder from the structure, will result in significantly lower component costs than counterparts made by salt replication. The balance of good performance and good manufacturability demonstrated herein, coupled with low predicted costs for raw materials and manufacturing, highlights the scope for SMFs containing weak EGP to be researched and developed more widely.

\section{Acknowledgements}

This research did not receive any specific grant from funding agencies in the public, commercial, or not-for-profit sectors.

Author Contributions: Kennedy: Conceptualization, Methodology, Investigation, Writing- Original draft preparation, Formal analysis, Supervision. Cheneler: Software, Investigation, Data curation, Validation, Formal analysis, Writing- Reviewing and Editing,

\section{Data Availability}

The raw/processed data required to reproduce these findings cannot be shared at this time as the data also forms part of an ongoing study.

\section{References}

1. M.F. Ashby et al., Metal Foams: A Design Guide, Butterworth-Heinemann, Burlington, 2000

2. Banhart, J., “Aluminum Foams:," On the Road to Real Applications, vol. 04, no. 28, pp. 290-295, 2003.

3. Francisco García-Moreno, Commercial Applications of Metal Foams: Their Properties and Production. Materials 2016, 9, 85; doi:10.3390/ma9020085

4. John Banhart, Manufacture, characterisation and application of cellular metals and metal foams, Progress in Materials Science 46 (2001) 559-632

5. Jinnapat, A., Kennedy, A. The Manufacture and Characterisation of Aluminium Foams Made by Investment Casting Using Dissolvable Spherical Sodium Chloride Bead Preforms. Metals 2011, 1, 49-64. 
6. Russell Goodall, Jean-Francois Despois, Ariane Marmottant, Luc Salvo, Andreas Mortensen, The effect of preform processing on replicated aluminium foam structure and mechanical properties, Scripta Materialia 54 (2006) 2069-2073

7. Elizondo Luna, E. M., Barari, F., Woolley, R., Goodall, R. Casting Protocols for the Production of Open Cell Aluminum Foams by the Replication Technique and the Effect on Porosity.J. Vis. Exp.(94), e52268, doi:10.3791/52268 (2014).

8. Langston, P., Kennedy, A.R. Discrete element modelling of the packing of spheres and its application to the structure of porous metals made by infiltration of packed beds of $\mathrm{NaCl}$ beads,2014, Powder Technology,268,210-218

9. Wright, A. and Kennedy, A. (2017), The Processing and Properties of Syntactic Al Foams Containing Low Cost Expanded Glass Particles. Adv. Eng. Mater., 19: n/a, 1600467. doi:10.1002/adem.201600467

10. L.P. Zhang, Y.Y. Zhao, Mechanical response of Al matrix syntactic foams produced by pressure infiltration casting, J. Compos. Mater., 2007 41, 2105

11. J. Cox, D. D. Luong, V. C. Shunmugasamy, N. Gupta, O. M. Strbik III and K. Cho, Compressive Behavior and Microstructural Characteristics of Iron Hollow Sphere Filled Aluminum Matrix Syntactic Foams, Metals, 2014, 4, 530

12. A Rabiei, L, J Vendra, , A comparison of composite metal foam's properties and other comparable metal foams, Materials Letters 63 (2009) 533-536

13. Mohammed Yaseer Omar, Chongchen Xiang, Nikhil Gupta, Oliver M Strbik III, Kyu Cho, Syntactic foam core metal matrix sandwich composite: Compressive properties and strain rate effects, Materials Science \& Engineering, A643 (2015) 156-168

14. Mohammed Yaseer Omar, Chongchen Xiang, Nikhil Gupta, Oliver M. Strbik III, Kyu Cho Syntactic foam core metal matrix sandwich composite under bending conditions, Materials and Design, 86 (2015) $536-544$.

15. Rohatgi, P.K., Gupta, N., Schultz, B.F.et al. The synthesis, compressive properties, and applications of metal matrix syntactic foams. JOM 63, 36-42 (2011).

16. M. Altenaiji, G. K. Schleyer, Y. Y. Zhao, in Composites and Their Properties (Ed.: Ning Hu), InTech, Rijeka, Croatia 2012, p. 437.

17. J. Cox, D. D. Luong, V. C. Shunmugasamy, N. Gupta, O. M. Strbik III, K. Cho, Metals 2014, 4, 530.

18. D. K. Balch, J. G. O. Dwyer, G. R. Davis, C. M. Cady, G. T. Gray III, D. C. Dunand, Mater. Sci. Eng. A 2005, A391, 408.

19. G. H. Wu, Z. Y. Dou, D. L. Sun, L. T. Jiang, B. S. Ding, B. F. He, Scr. Mater. 2007, 56, 221.

20. Omya, "Product Information: Glass Ceramics, EGP40 [Technical Data]," private communication with supplier, 2015.

21. Equipment data: Per-Cast vacuum caster: https://www.hswalsh.com/product/cast-investment-andvacuum-casting-unit-tc083 accessed 27/4/2020

22. Product data LM6 alloy, Norton Aluminium, http://www.nortal.co.uk/LM6/ accessed 29/11/2019.

23. Otaru, AJ, Kennedy, AR, 2019, 'Investigation of the Pressure Drop Across Packed Beds of Spherical Beads: Comparison of Empirical Models With Pore-Level Computational Fluid Dynamics 
Simulations',Journal of Fluids Engineering, Transactions of the ASME, vol. 141, no. 7, 071305.https://doi.org/10.1115/1.4042957

24. Jia, J., Siddiq, A.R., Kennedy, A.R. Porous titanium manufactured by a novel powder tapping method using spherical salt bead space holders: Characterisation and mechanical properties, 2015, Journal of the Mechanical Behavior of Biomedical Materials,48,229-240

25. Siddiq, A.R., Kennedy, A.R. Porous poly-ether ether ketone (PEEK) manufactured by a novel powder route using near-spherical salt bead porogens: Characterisation and mechanical properties, 2015, Materials Science and Engineering C47,180-188

26. Jinnapat, A.R. Kennedy, The manufacture of spherical salt beads and their use as dissolvable templates for the production of cellular solids via a powder metallurgy route, Journal of Alloys and Compounds, Volume 499, Issue 1, 4 June 2010, Pages 43-47

27. A. Mortensen, Y. Conde, A. Rossoll, C. San Marchi, Scaling of conductivity and Young's modulus in replicated microcellular materials, J. Mater. Sci. 48 (23) (2013) 8140-8146.

28. Bowen, J., Cheneler D., "Closed-Form Expressions for Contact Angle Hysteresis: Capillary Bridges between Parallel Platens" Colloids Interfaces, 2020, 4(1), 13.

29. Ying Cheng, Yanxiang Li, Xiang Chen, Xu Zhou, and Ningzhen Wan, Compressive Properties and Energy Absorption of Aluminum Foams with a Wide Range of Relative Densities, JMEPEG (2018) 27:4016-4024

30. Kadhim Al-Sahlani, Steffen Broxtermann, Daniel Lell, Thomas Fiedler, Effects of particle size on the microstructure and mechanical properties of expanded glass-metal syntactic foams, Materials Science and Engineering: A, Volume 728, 13 June 2018, Pages 80-87.

31. Kadhim Al-Sahlani, Mehdi Taherishargh, Erich Kisi and Thomas Fiedler, Controlled Shrinkage of Expanded Glass Particles in Metal Syntactic Foams, Materials 2017, 10, 1073; doi:10.3390/ma10091073

32. J.F.Despois, A.Marmottant, L.Salvo, A.Mortensen, Influence of the infiltration pressure on the structure and properties of replicated aluminium foams, Materials Science and Engineering: A, Volume 462, Issues 1-2, 2007, Pages 68-75.

33. C. San Marchi, A. Mortensen, Deformation of open-cell aluminium foam, Acta mater. 49 (2001) 39593969.

34. E.Hernández-Nava, C.J.Smith, F.Derguti, S.Tammas-Williams, F.Léonard, P.J.Withers, I.Todd, R.Goodall, Acta Materialia, The effect of density and feature size on mechanical properties of isostructural metallic foams produced by additive manufacturing, Volume 85, 2015, Pages 387-395.

35. L. Xi, P. Wang, K.G. Prashanth, H. Li, H.V. Prykhodko, S. Scudino, I. Kaban. "Effect of $\mathrm{TiB}_{2}$ particles on microstructure and crystallographic texture of Al-12Si fabricated by selective laser melting." Journal of Alloys and Compounds 786 (2019): 551-556

36. Cheneler, D., A. R. Kennedy. "Measurement and modelling of the elastic defection of novel metal syntactic foam composite sandwich structures in 3-point bending." Composite Structures (2019): 111817. 